\title{
EDITORIAL
}

\section{Idiopathic pulmonary fibrosis trials: recommendations for the jury}

\author{
S.D. Nathan* and R.M. du Bois"
}

I diopathic pulmonary fibrosis (IPF) is a disease with a dismal prognosis and a median survival of only $2-3$ yrs. There are no licensed medical therapies in the USA and the only care options that are endorsed by the most recent consensus guidelines are lung transplantation and enrolment in a clinical trial [1]. Although the past decade has seen numerous trials in IPF being undertaken and completed, the outcomes have mostly been disappointing with only one or two exceptions [2, 3]. Despite this, valuable insights about the natural history and course of the disease have been gained from the wellcharacterised patient data sets of these trials. However, these studies have raised many questions and fuelled the continuing debate on how future IPF trials should be designed. There are many considerations and challenges involved in the implementation and completion of IPF studies. The complex nature of the pathogenic process, the highly variable disease course, the possibility of inadequate drug deposition in the targeted area and the uncertainty about the most appropriate end-points may all confound the interpretation of clinical trial results.

\section{THE NATURE OF THE PATHOGENIC PROCESS}

It is believed that IPF is the result of derangements of the alveolar epithelial membrane followed by an inappropriate wound healing response, for reasons that remain unknown. These perturbations occur at unpredictable intervals with great inter-individual variability over many years. Therefore, at clinical presentation, the pathology consists of a combination of relatively unaffected areas, together with more advanced, established fibrosis and various degrees of histopathology between these extremes. Each stage of this dynamic disease process is marked by simultaneous cellular derangements, activated pathways and dysregulated cytokines co-localising with upstream initiators and downstream consequences. This intricate network of mediators and pathways may be accompanied by autocrine and escape pathways that limit the ability of a unimechanistic agent to thwart the disease. To impact on this complex pathogenic process, the ideal drug should, therefore, be pleiotropic in its actions, with the ultimate management probably entailing a cocktail of therapies.

A major therapeutic challenge is how to interrupt those pathways that are driving disease without inappropriately

*Advanced Lung Disease and Lung Transplant Program, Inova Fairfax Hospital, Falls Church, VA, USA. " Imperial College, London, UK.

CORRESPONDENCE: S.D. Nathan, Advanced Lung Disease and Transplant Program, Inova Heart and Vascular Institute, 3300 Gallows Road, Falls Church, VA 22042, USA. E-mail: steven.nathan@inova.org hindering those mechanisms that are striving, although failing, to produce homeostasis. Indeed, is such precise targeting achievable in the context of the repetitive, temporally disparate initiating insult and subsequent pathobiology that produces a variety of histopathological stages at all time-points?

\section{CHARACTERISTICS AND COURSE OF THE DISEASE}

There is a growing appreciation that several distinct phenotypes exist within what is currently regarded as IPF. It is conceivable that the efficacy of any given therapy might similarly be variable, based on the unique phenotypic characteristics and the stage of the disease. Indeed, the heterogeneous nature of the disease and probable varied response to different therapies might partially explain the negative results and mixed signals seen from many of the prior drug studies. Clinical variants include a temporal spectrum of disease progression that varies from those individuals with a slowly protracted course, to patients with a more rapid pattern of progression, including those who are predisposed to unpredictable acute exacerbations [4-6]. Radiographically, it does appear that there are distinctive high-resolution computed tomography patterns that make the identification of patients with varying prognoses possible [7-9]. Whether these disease variants have a differential treatment response remains to be determined and may require stratification at baseline in future therapeutic trials.

In the context of a therapy that has some effect, phenotypic variability is likely to contribute to a nonuniform response in the treatment arm, as well as influence disease behaviour in the placebo arm. If some individuals in each group have relatively stable disease, then attenuation in decline cannot be demonstrated. Therefore, the average difference in change in the endpoint measure will be smaller than if all patients in both groups declined during the course of the trial. Enriching study populations by only including those with an a priori higher risk of progression and mortality has obvious appeal in powering the end-point. However, there is inherent difficulty in the upfront recognition of the projected pattern of progression for individual patients. Emerging evidence suggests that genomic signature characterisation may enable the identification of patients with varying disease patterns of progression [10-12]. With further validation and broader availability, such categorisation might find utility in homogenising study populations in future clinical trials.

\section{THERAPEUTIC CONSIDERATIONS}

In the context of the complexities in IPF pathogenesis, the ideal agent should target a redundancy of mechanisms. Indeed, there 
are data supporting the concept that therapies that have demonstrated some evidence of efficacy are multi-mechanistic in their action $[2,3]$. Further issues pertaining to the nature of possible novel drugs include the appropriate dose and frequency of administration, the duration of therapy needed to demonstrate an effect and the correct mode of delivery to ensure adequate drug deposition at the actionable site. Specifically, for any agent to exert its effects, the concept of matching drug deposition to disease distribution requires reconciliation. Although neovascularisation is well described in IPF, a predominance of vascular ablation is known to characterise the disease. An attractive theoretical therapeutic approach is to identify areas of disease where the drug may be most deliverable and target the cells or pathways that might interrupt the disease in these regions. For example, increased vascularity has been demonstrated peripheral to the fibroblastic focus, which might allow adequate levels of systemically delivered drug to deposit in these areas, thus targeting the arrest of these cells in an early stage of their differentiation [13].

To date, most IPF studies have included orally or subcutaneously administered medications. Drug delivery via the inhaled route has appeal, as this might allow higher concentrations of active drugs to reach sites of disease activity and enable drugs with poor oral bioavailability or avid hepatic degradation to be subjected to study. This route of administration might also allow higher doses to be employed with fewer systemic side-effects.

\section{WHICH OUTCOME MEASURES ARE CLINICALLY RELEVANT?}

The ideal measure should be reproducible, responsive, valid and easy to measure. It should also be equally applicable to all IPF phenotypes included in the study. Changes in the chosen measure should be clinically meaningful, such that the result of the clinical trial will have unequivocal patient management implications. Unfortunately, no consensus or consistency exist amongst currently reported studies with regard to the optimal outcome measures. Commonly used end-points in the prospective IPF studies to date have included: change in forced vital capacity (FVC); diffusing capacity of the lung for carbon monoxide (DL,CO); 6-min walk test distance (6MWT) or oxygen desaturation; hospitalisation-free period; time to clinical worsening; and survival.

Clearly, the only indisputable end-point would appear to be mortality, but survival alone as the primary end-point is also laden with inherent difficulties. Specifically, the number of patients and study duration required for adequate power may be prohibitive. In this context, it is generally accepted that if a drug is to impact on mortality, then the earlier it is started, the greater the likelihood of success. However, with "early" IPF patients, the mortality rate is likely to be initially low and thus patients would need to be retained and followed for a number of years. The duration of most of the studies to date has been in the range of 6 months to 2 yrs with drop-out rates ranging from 1 to $41 \%[2,14]$. For studies that extend beyond 2 yrs, the drop-out rate will become more problematic, as might the notion of patients dying with their IPF rather than from their IPF. Therefore, mortality as the primary end-point might be best suited for studies tailored to those with advanced disease.

Of all the surrogate end-points proposed or employed to date, change in FVC has been the most commonly utilised since it most closely fulfils the ideal characteristics. Nonetheless, how best to evaluate change in this (as well as other) end-point measurements requires closer scrutiny. Outcome measures can be broadly classified as those that involve continuous variables or categorical "events". When a categorical end-point analysis is being contemplated, an important consideration in selecting the outcome measure is to establish a clinically meaningful threshold which either represents the minimally clinical important difference (MCID) or imparts important prognostic information. A change in FVC of $>10 \%$ has been used in most studies, but recent data suggest that a change as small as 5\% might also have significant prognostic implications [15]. Although the 6MWT is less well validated in IPF, there are now data that support an MCID of $24-45 \mathrm{~m}$, and a change of $>50 \mathrm{~m}$ has been shown to be associated with a four-fold relative risk for mortality [15]. A third approach to the serial analysis of continuous physiological measures is to evaluate the slope of change. The value of such an analysis is that all measures during the course of the study influence the result, rather than a measure at one arbitrary time-dependent point. The heterogeneity of progression in individual patients may be better captured through this approach.

The optimal study duration is also unknown and the current inability to reconcile this makes event-driven studies an attractive option. The event rate is the major determinant of the anticipated study population size and the duration of the trial. Enriching the patient population with those who are most likely to have the "event" may enable the timely, successful completion of the study. However, potential limitations include the a priori identification of at-risk patients and having an exclusive study population, which then limits the broader subsequent applicability of the results.

Enabling the event rate through the implementation of a composite primary end-point is another possibility. Composite end-points should be constituted by quantifiable measures that reflect a spectrum of pathophysiological consequences of disease progression [16]. However, what constitutes an accepted, validated composite end-point in IPF is, at present, unknown. Change in FVC and distance walked in 6 min have both been proven to be robust predictors of outcomes as single measures $[15,17,18]$. They may be sufficiently distinct in their respective physiological domains that the two in combination have appeal as a composite measure. Other potential parameters for inclusion in a composite index are changes in $D \mathrm{~L}, \mathrm{CO}$, quality of life, respiratory hospitalisations or acute exacerbations, lung transplantation and death. Some of these have their own inherent drawbacks, for example, the threshold for transplantation and time on the transplant waiting list differs significantly both nationally and regionally, as does the threshold for hospitalisation. The ideal composite should be constituted by parameters or events that are of equal importance, comparable frequency and similar sensitivity to the therapeutic intervention [16]. However, these idealistic criteria are rarely satisfied and results based on composite end-points run the risk of being misleading when they are powered by the most frequent, but least important, of its constituents. An option to address this issue of imbalance is through the implementation of weighted composite end-points. This approach enables equipoise by handicapping the relative importance of the components of the composite [16]. Individual analysis of the components should also be built into the study 
design. These should all at least point in the direction of efficacy and thereby serve as a validation of the composite.

\section{CONCLUSION}

Pathophysiological disease-specific hurdles and uncertainty with regards to the best end-point(s), as well as the difficulty of performing mortality studies, have hampered the discovery of universally applicable therapies for IPF. The value of prior IPF studies lies not only in providing new information about the natural history of the disease, but also in guiding subsequent study designs. Future approaches should include the ongoing evaluation of currently available agents alone and in combination, the identification of novel drugs with pleiotropic actions, and trials with validated, weighted composite end-points. Further genomic signature differentiation and validation may, in the future, enable study enrichment with those patients at highest risk of progression and mortality.

\section{STATEMENT OF INTEREST}

Statements of interest for both authors can we found at www.erj. ersjournals.com/site/misc/statements.xhtml

\section{REFERENCES}

1 Raghu G, Collard HR, Egan JJ, et al. An Official ATS/ERS/JRS/ ALAT Statement: idiopathic pulmonary fibrosis: evidence-based guidelines for diagnosis and management. Am J Respir Crit Care Med 2011; 183: 788-824.

2 Demedts M, Behr J, Buhl R, et al. High-dose acetylcysteine in idiopathic pulmonary fibrosis. N Engl J Med 2005; 353: 2229-2242.

3 Taniguchi H, Ebina M, Kondoh Y, et al. Pirfenidone in idiopathic pulmonary fibrosis. Eur Respir J 2010; 35: 1-9.

4 Nathan SD, Shlobin OA, Weir N, et al. Long-term course and prognosis of idiopathic pulmonary fibrosis in the new millennium. Chest 2011; 140: 221-229.

5 Song JW, Hong S-B, Lim C-M, et al. Acute exacerbation of idiopathic pulmonary fibrosis: incidence, risk factors and outcome. Eur Respir J 2011; 37: 356-363.
6 Nishiyama O, Taniguchi $\mathrm{H}$, Kondoh $\mathrm{Y}$, et al. A simple assessment of dyspnoea as a prognostic indicator in idiopathic pulmonary fibrosis. Eur Respir J 2010; 36: 1067-1072.

7 Flaherty KR, Thwaite EL, Kazerooni EA, et al. Radiological versus histological diagnosis in UIP and NSIP: survival implications. Thorax 2003; 58: 143-148.

8 Cottin V, Nunes H, Brillet PY, et al. Combined pulmonary fibrosis and emphysema: a distinct underrecognised entity. Eur Respir J 2005; 26: 586-593.

9 Tcherakian C, Cottin V, Brillet PY, et al. Progression of idiopathic pulmonary fibrosis: lessons from asymmetrical disease. Thorax 2011; 66: 226-231.

10 Selman M, Carrillo G, Estrada A, et al. Accelerated variant of idiopathic pulmonary fibrosis: clinical behaviour and gene expression pattern. PLoS ONE 2007; 2: e482.

11 Trujillo G, Meneghin A, Flaherty KR, et al. TLR9 differentiates rapidly from slowly progressing forms of idiopathic pulmonary fibrosis. Sci Transl Med 2010; 2: 1-10.

12 Konishi K, Gibson KF, Lindell KO, et al. Gene expression profiles of acute exacerbations of idiopathic pulmonary fibrosis. Am J Respir Crit Care Med 2009; 180: 167-175.

13 Renzoni EA, Walsh DA, Salmon M, et al. Interstitial vascularity in fibrosing alveolitis. Am J Respir Crit Care Med 2003; 167: 438-443.

14 King TE, Albera C, Bradford WZ, et al. Effect of interferon gamma$1 \mathrm{~b}$ on survival in patients with idiopathic pulmonary fibrosis (INSPIRE): a multicentre, randomized, placebo-controlled trial Lancet 2009; 374: 222-228.

$15 \mathrm{du}$ Bois RM, Weycker D, Albera C, et al. 6-minute walk test in idiopathic pulmonary fibrosis: test validation and minimal clinically important difference. Am J Respir Crit Care Med 2011; 183: 1231-1237.

16 Kaul S, Diamond GA. Trial and error: how to avoid commonly encountered limitations of published clinical trials. J Am Coll Cardiol 2010; 55: 415-427.

17 Zappala CJ, Latsi PI, Nicholson AG, et al. Marginal decline in forced vital capacity is associated with a poor outcome in idiopathic pulmonary fibrosis. Eur Respir J 2010; 35: 830-836.

18 du Bois RM, Albera C, Bradford WZ, et al. Ascertainment of individual risk of mortality for patients with idiopathic pulmonary fibrosis (IPF). Am J Respir Crit Care Med 2010; 181: A2499. 